\title{
Cardiovascular risks in type 2 diabetes and the interpretation of cardiovascular outcome trials
}

This article was published in the following Dove Medical Press journal: Diabetes, Metabolic Syndrome and Obesity:Targets and Therapy

\section{Deborah Hinnen' \\ Davida F Kruger ${ }^{2}$}

'Department of Health and Wellness, Memorial Hospital Diabetes Center, University of Colorado Health, Colorado Springs, CO 80909, USA; ${ }^{2}$ Henry Ford Health System, Division of Endocrinology, Diabetes, Bone and Mineral Disease, Henry Ford Medical Center - New Center One, Detroit, MI 48202, USA
Correspondence: Deborah Hinnen Memorial Hospital Diabetes Center, University of Colorado Health, $175 \mathrm{~S}$ Union Blvd Ste 305, Colorado Springs, CO 80909 , USA

$\mathrm{Tel}+\mathrm{I} 719365688$ I

Email Deborah.Hinnen@uchealth.org
Background: Patients with type 2 diabetes (T2D) are at increased cardiovascular (CV) risk compared to subjects without diabetes, with some data estimating that CV disease (CVD) risk is doubled in these individuals. Additionally, CVD remains the leading cause of death in patients with T2D, so it is paramount to determine the relationship between these two diseases.

Purpose: Older diabetes treatments have limited CV safety data. In 2008, the US Food and Drug Administration published guidance for manufacturers on antihyperglycemic agents, requiring studies to ensure CV safety of new therapies. Since then, manufacturers of many newer agents have conducted and published results from CV outcomes trials (CVOTs), with more trials due to publish soon. This review discusses the relationship between CVD and T2D and explores findings from the latest CVOTs of glucose-lowering agents to guide nurse practitioners in their prescribing patterns for patients with T2D.

Conclusion: Patients with T2D are at high risk of CVD, so CV risk should be carefully considered when managing these patients, and CV risks and benefits of antidiabetic drugs should be included in prescribing decisions.

Keywords: cardiovascular disease, chronic disease management, type 2 diabetes mellitus, nurse practitioners

\section{Overview of the relationship between cardiovascular risk and type $\mathbf{2}$ diabetes}

Prior to the advent of insulin therapy, limited tools were available to prevent the progression of diabetes. As patient survival increased with insulin use, cardiovascular (CV) disease (CVD) management emerged as a key priority in improving patient lives. ${ }^{1}$ Patients with type 2 diabetes (T2D) are still at increased CV risk compared to their peers without diabetes, with some data suggesting that they are at double the risk of CVD compared to their healthy counterparts. ${ }^{1-3} \mathrm{CVD}$ also remains the leading cause of death in T2D. ${ }^{4,5}$

A scientific statement from the American Heart Association and the American Diabetes Association (ADA) explains that for every $18 \mathrm{mg} / \mathrm{dL}(1 \mathrm{mmol} / \mathrm{L})$ increase in fasting plasma glucose, the risk of future $\mathrm{CV}$ events or death increases by $17 \% .{ }^{2} \mathrm{~A}$ rise of $1 \%(11 \mathrm{mmol} / \mathrm{mol})$ in $\mathrm{HbA}_{1 \mathrm{c}}$ results in an $18 \%$ increase in risk of $\mathrm{CV}$ events. ${ }^{2}$ Furthermore, an analysis of eleven large, integrated health care organizations in the United States found an increased CV risk if $\mathrm{HbA}_{1 \mathrm{c}}$ was $\geq 9 \%(75 \mathrm{mmol} / \mathrm{mol}){ }^{6}{ }^{6}$ The European Prospective Investigation of Cancer and Nutrition (EPIC)-Norfolk study found that an increase of $1 \%$ in $\mathrm{HbA}_{1 \mathrm{c}}$ is associated with a $28 \%$ increase in risk of all-cause death in men, independent of age, blood pressure, serum cholesterol, body mass index, and cigarette smoking $(P<0.002){ }^{7}$ submit your manuscript Dovepress if in $>$ 
Data from the UKPDS trial in patients with T2D without CVD demonstrated a reduction of at least $12 \%$ in the risk of $\mathrm{CV}$ complications with every $1 \%$ decrease in $\mathrm{HbA}_{10},{ }^{8}$ as well as a reduction in the risk of myocardial infarction (MI) with intensive vs standard therapy after 10 -year observation. ${ }^{9}$ According to the ADA/European Association for the Study of Diabetes (EASD) Position Statement 2015, the impact of glucose control on CV complications remained to be fully elucidated at that time; a benefit may only emerge after many years of improved glycemic control. ${ }^{10}$ Consequently, the ADA and EASD have recently released a joint consensus report to aid the decision-making process when prescribing antidiabetic therapies, with emphasis being placed on the management of $\mathrm{CV}$ risk factors. ${ }^{11}$

This review will discuss the relationship between CVD and T2D and examine the findings from the latest CV outcomes trials (CVOTs) of glucose-lowering agents.

\section{Cardiovascular outcomes trials in type 2 diabetes}

Historically, new therapies for T2D required evidence for improving glycemic control from Phase II and III trials. However, in 2008, after the controversial results from a meta-analysis of rosiglitazone trials, ${ }^{12}$ the US Food and Drug Administration published "Guidance for industry", stating that manufacturers of all new antidiabetic therapies must also demonstrate that the therapy will not result in an increased risk for CVD. ${ }^{13}$ Since this guidance was produced, a number of CVOTs have been conducted, to ensure that antidiabetic agents do not increase the risk of CV events. In CVOT design, a glucose-lowering drug is added to standard of care $(\mathrm{SoC})$ treatments in patients at high risk of $\mathrm{CV}$ events (either patients with advanced diabetes, elderly patients, or those with renal impairment), and compared with $\mathrm{SoC}$ alone or added to an active comparator. The majority of CVOTs use a composite primary outcome known as a 3-point major adverse cardiovascular event (MACE), comprising $\mathrm{CV}$ death, nonfatal MI, and nonfatal stroke. Some also include hospitalization for heart failure (HF) and/or other endpoints. ${ }^{13}$ According to the guidance, CVOTs comparing an antihyperglycemic agent with a comparator must demonstrate that the upper bound of a two-sided $95 \% \mathrm{CI}$ is $<1.8 .{ }^{13}$ Recently, results from these CVOTs have demonstrated that some newer glucose-lowering therapies appear not only to have no detrimental impact on CV safety but additionally have beneficial effects on CVD beyond simple glucose control. ${ }^{14-16}$

\section{Review of the cardiovascular safety profile of medications for type 2 diabetes}

All manufacturers of dipeptidyl peptidase 4 inhibitors (DPP4is), sodium-glucose cotransporter-2 inhibitors (SGLT-2is), and glucagon-like peptide-1 receptor agonists (GLP-1RAs) have initiated CVOTs, many of which are complete, and there are a number of other drugs with demonstrable CV safety (Table 1). Many older drugs do not have published CVOTs, but some have established CV outcomes from other trials (Table 1).

Metformin, sulfonylureas, thiazolidinediones, meglitinides, and alpha-glucosidase inhibitors

Metformin remains the mainstay of first-line therapy in the treatment of $\mathrm{T}_{2} \mathrm{D}^{17}$ owing to its efficacy in lowering glucose, neutral effect on weight, minimal hypoglycemia, and low cost. Metformin has also been shown to reduce the risk of CV outcomes, including MI, HF, and stroke. ${ }^{18}$ Sulfonylureas (SUs) appear to have mixed effects on CV endpoints. A meta-analysis of randomized controlled trials with SUs demonstrated no significant effects on MACE or MI independently, but an association with an increased risk of stroke and CV death was observed. ${ }^{19}$ The thiazolidinedione (TZD) rosiglitazone is associated with an increased risk of MI and CV mortality. ${ }^{12}$ Despite later data from the RECORD trial showing no increased risk in overall $\mathrm{CV}$ morbidity and mortality ${ }^{20}$ (although it did show an increase in HF), it is generally well accepted that the initial results from the meta-analysis are more reliable than RECORD, as this trial was unblinded..$^{21,22}$ Conversely, patients treated with another TZD, pioglitazone, experienced a reduction in the progression of atherosclerosis, significantly reduced plasma lipid levels compared with rosiglitazone, and decreased apoptosis induced by ischemic injury, suggesting that the $\mathrm{CV}$ effects of TZDs are not a class effect. ${ }^{23}$ The CV safety of meglitinides is yet to be fully elucidated, ${ }^{24}$ although the NAVIGATOR study demonstrated similar rates in CV outcomes in patients treated with nateglinide compared with those treated with placebo. ${ }^{25}$ Data are also lacking on the CV safety of alphaglucosidase inhibitors, although the Acarbose Cardiovascular Evaluation (ACE) trial demonstrated no reduction in MACE in Chinese patients with impaired glucose tolerance treated with acarbose. ${ }^{26}$

\section{The clinical implications of CVOT data for older antidiabetic therapies}

Although metformin has the longest history of CV safety in antidiabetic therapies, there are no modern CVOTs to assess 


\begin{tabular}{|c|c|c|c|c|c|c|c|}
\hline & 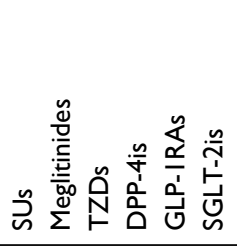 & & & 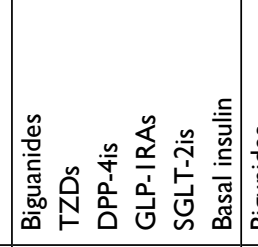 & 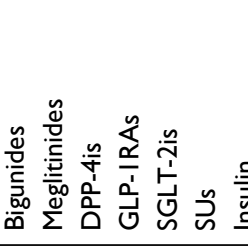 & 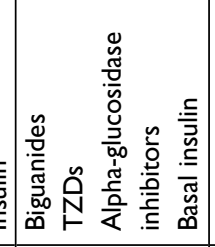 & 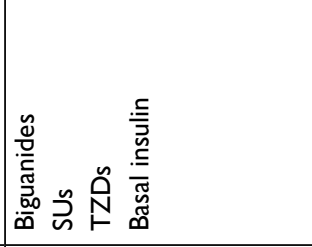 \\
\hline 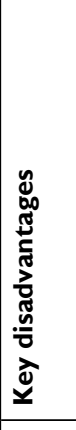 & 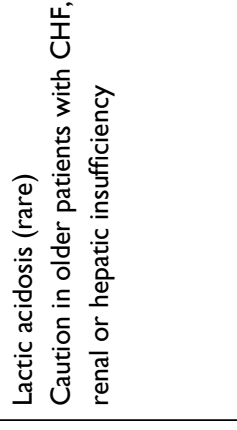 & 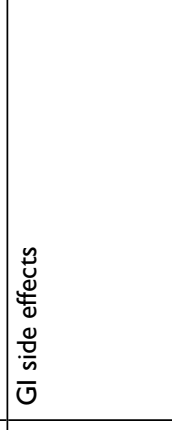 & 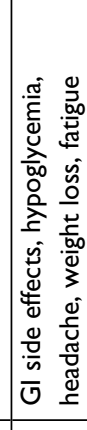 & 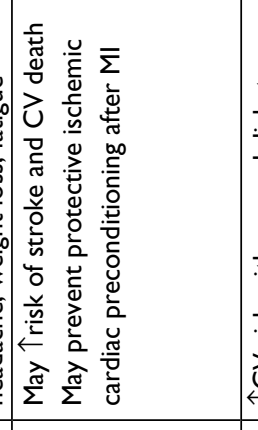 & 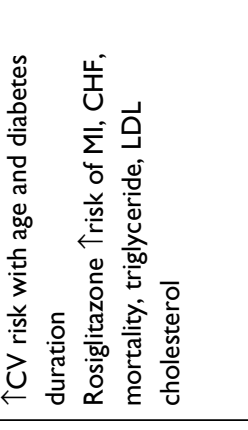 & 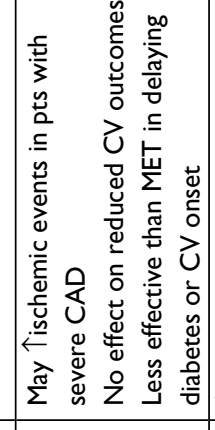 & 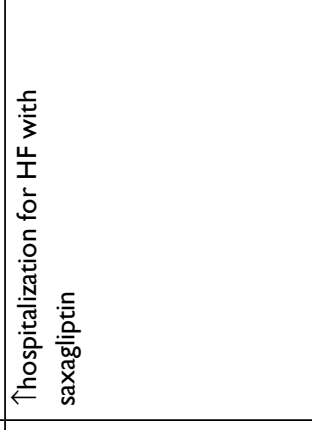 \\
\hline 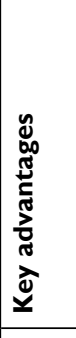 & 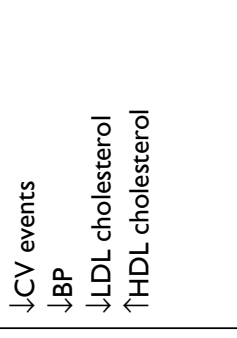 & 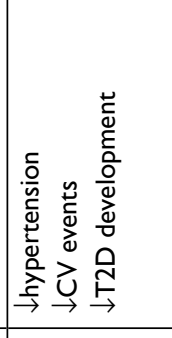 & 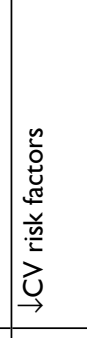 & 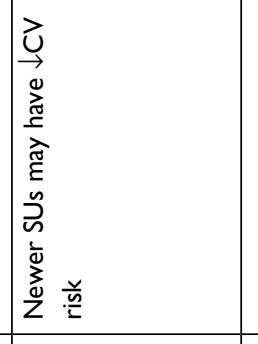 & 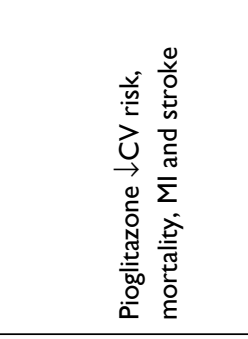 & 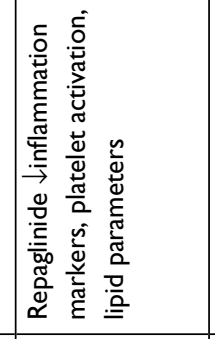 & 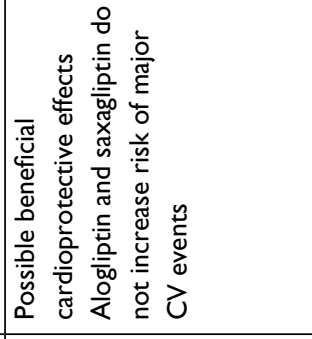 \\
\hline 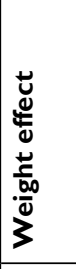 & 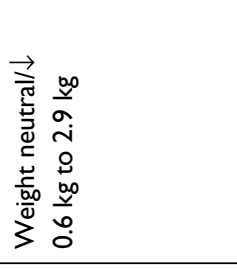 & 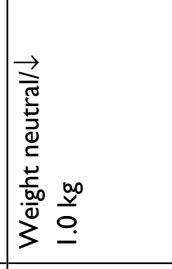 & 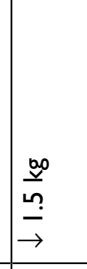 & 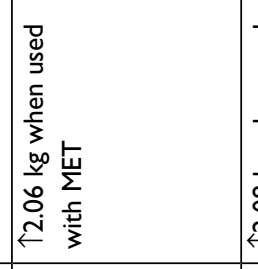 & 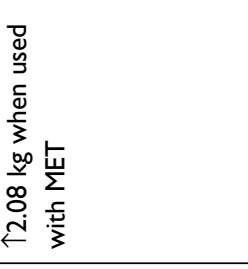 & 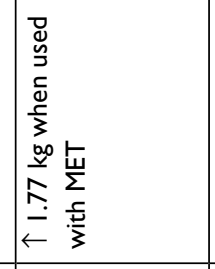 & 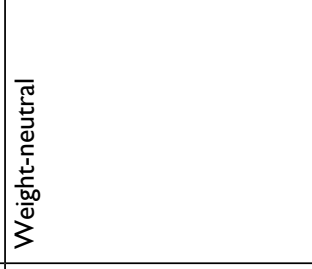 \\
\hline 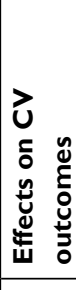 & $\frac{\mathscr{J}}{E}$ & 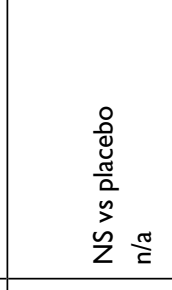 & $\stackrel{\frac{\pi}{E}}{E}$ & 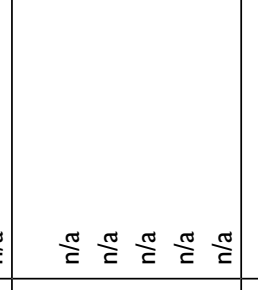 & 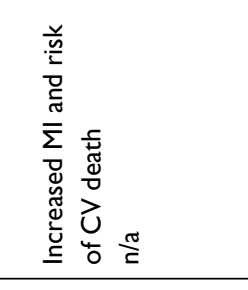 & $\stackrel{\mathbb{E}}{E}$ & 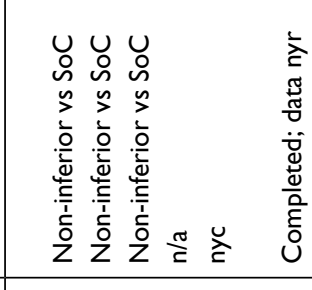 \\
\hline 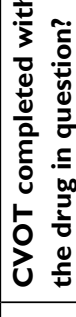 & $\stackrel{\circ}{z}$ & 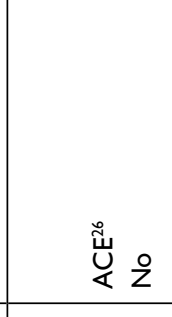 & $\stackrel{\circ}{2}$ & 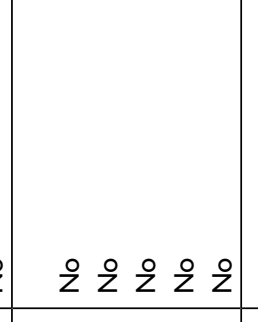 & 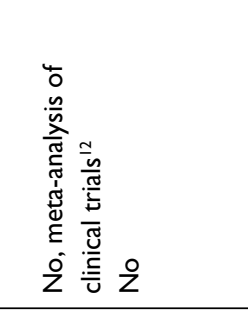 & z을 & 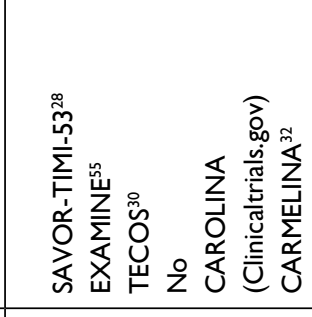 \\
\hline 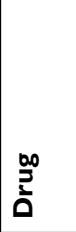 & 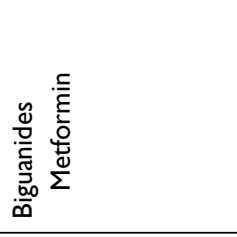 & 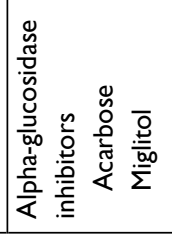 & & 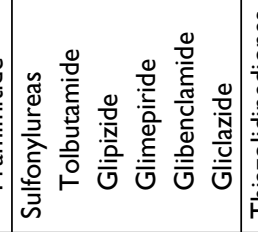 & 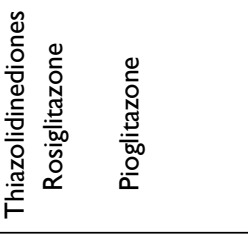 & 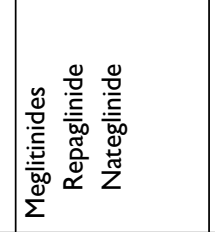 & 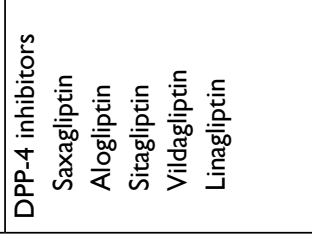 \\
\hline
\end{tabular}




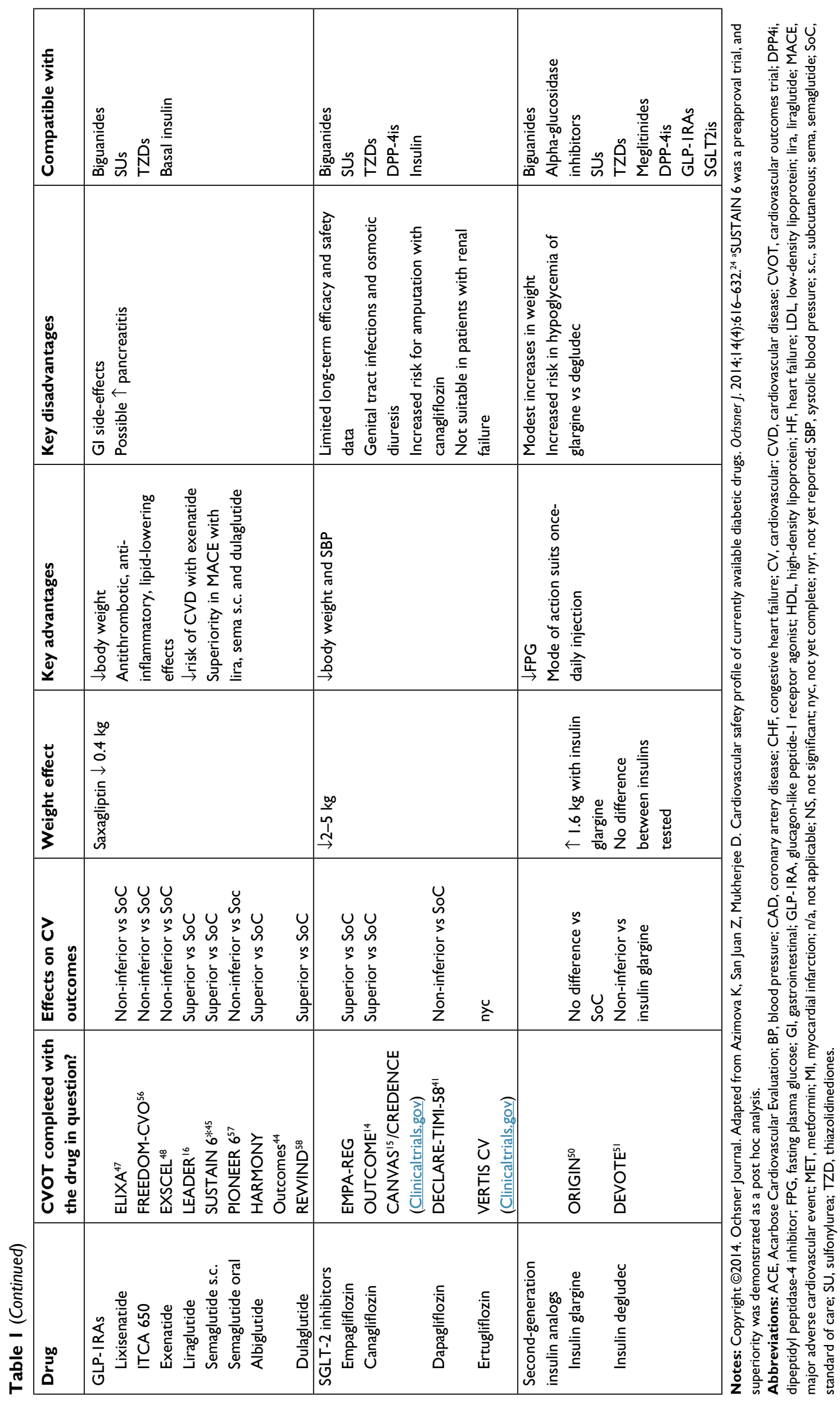


benefits and safety of metformin on a background of current SoC for glucose control and other CV risk factors. Treatment with SUs is associated with weight gain, which remains a concern in patients with T2D. Moreover, an increased possibility of $\mathrm{CV}$ death and stroke means that prescribers should probably avoid these agents in patients at high risk for these events. Furthermore, rosiglitazone has had mixed $\mathrm{CV}$ results, ${ }^{12,20}$ indicating its use in patients at high risk for CV events needs to be considered carefully.

\section{Newer therapies for T2D: dipeptidyl peptidase-4 inhibitors (DPP-4is), sodium-glucose cotransporter-2 inhibitors (SGLT-2is), glucagon-like peptide- I receptor agonists (GLP-IRAs), and second- generation insulin analogs}

A comprehensive meta-analysis evaluating data from 170 randomized controlled trials comparing the $\mathrm{CV}$ effects of novel antidiabetic medications (including DPP-4is, GLP-1RAs, and SGLT-2is) with those of traditional classes of drugs (including insulin, metformin, SU, and TZD) determined that, in general, newer antidiabetic agents show favorable CV safety, yet discrepancies exist between individual and class comparisons. ${ }^{27}$

To date, all CVOTs of DPP-4is have reported noninferiority in terms of $\mathrm{CV}$ safety with the composite MACE endpoint when compared with placebo, both on a background of SoC, thus suggesting no harm. ${ }^{28-30}$ Results from a recently published meta-analysis also confirmed a lack of CV benefit and inferred that DPP-4is are inferior to SGLT-2is and GLP-1RAs in preventing CV-related death. ${ }^{31}$ The CAROLINA trial is yet to publish results for the CV safety of linagliptin (NCT01243424, Clinicaltrials.gov). However, preliminary findings from the CARMELINA trial suggest that treatment with linagliptin has no impact on $\mathrm{CV}$ and $\mathrm{HF}$ events. ${ }^{32}$

Notably, some negative individual CV outcomes have been revealed with specific DPP-4is. Saxagliptin has been associated with an increased risk for hospitalization for $\mathrm{HF}^{33}$ While alogliptin was not associated with increased risk of certain HF outcomes (such as hospital admission for HF) compared with placebo, hospital admissions for HF occurred significantly more often in patients without prior history of HF treated with alogliptin compared with placebo. ${ }^{34}$

Other DPP-4is have not been associated with an increased risk of HF. Both linagliptin and sitagliptin do not appear to increase the risk of HF in patients with diabetes. ${ }^{35}$ As these results are conflicting with those from saxagliptin and alogliptin, the HF concerns may not be a class effect, and currently, no mechanism for the association between DPP-4is and HF has been identified. ${ }^{35}$ It should be noted that the trial designs and patient population of these trials are not directly comparable, and therefore these safety differences may be attributable to these other factors rather than to the drugs.

Findings from two recent meta-analyses of data on SGLT-2is suggest favorable effects against CV outcomes and death. ${ }^{36,37}$ These reflect that the CVOT data for SGLT-2is empagliflozin and canagliflozin have demonstrated superiority of the trial drug in MACE outcomes when compared with SoC, suggesting a cardioprotective effect of these agents. ${ }^{14,15}$ Data from the EMPA-REG OUTCOME trial with empagliflozin led to an update in the label, and the therapy is now indicated to reduce the risk of $\mathrm{CV}$ death in patients with T2D and established CVD. ${ }^{38}$ This decision has since been validated by the ADA and EASD. ${ }^{11,39,40}$ The DECLARETIMI 58 Phase III CVOT with dapagliflozin demonstrated noninferiority, but not superiority, for MACE. ${ }^{41}$ When the components of this composite endpoint were analyzed, it was shown that dapagliflozin significantly reduced the number of hospitalizations for $\mathrm{HF}^{41}$ It seems unlikely therefore that the $\mathrm{CV}$ benefits associated with empagliflozin and canagliflozin are a class effect, but perhaps there is a class effect for HF. It is also unknown if empagliflozin, canagliflozin, or other SGLT-2is will have similar effects in patients with T2D but at low $\mathrm{CV}$ risk. ${ }^{40}$

Data from further CVOTs with ertugliflozin and sotagliflozin may help to elucidate if any SGLT-2i class effect exists (NCT01986881 and NCT03521934, respectively, Clinicaltrials.gov). The current ongoing VERTIS CV study assesses ertugliflozin in patients with T2D and established vascular disease, and the SOLOIST-WHF trial assesses sotagliflozin in patients with T2D with worsening HF. It will still be some time before the long-term safety and realworld impact of SGLT-2is in patients with T2D with HF/ CVD become clear.

Meta-analyses of CVOT data on GLP-1RAs suggest cardioprotective effects in patients with high $\mathrm{CV}$ risk. ${ }^{42,43} \mathrm{~A} \mathrm{CV}$ benefit was demonstrated in both the LEADER trial and the HARMONY Outcomes trial for the GLP-1RAs liraglutide and albiglutide, respectively, when compared with SoC. ${ }^{16,44}$ Also, even though testing for superiority of the primary composite endpoint was not prespecfied in SUSTAIN 6, a post hoc analysis of its data suggests that the once-weekly subcutaneous GLP-1RA, semaglutide, may also offer a CV benefit. ${ }^{45}$ In the LEADER trial, significantly fewer patients treated with liraglutide experienced MACE, compared with those 
treated with placebo, a statistically superior result. ${ }^{16}$ This led to an update in the label, and liraglutide is now indicated to reduce the risk of MACE in patients with T2D and established $\mathrm{CVD},{ }^{46}$ which is also reflected in the new consensus report from the ADA/EASD. ${ }^{11}$ The SUSTAIN 6 trial demonstrated noninferiority of subcutaneous semaglutide to placebo for MACE, and a post hoc analysis also showed superior CV benefit for patients treated with semaglutide compared with those treated with placebo. Testing for superiority was not prespecified or adjusted for multiplicity. ${ }^{45}$ In the HARMONY Outcomes trial, albiglutide therapy was found to be superior to placebo with respect to MACE and resulted in a significant reduction in MIs (it is important to note that albiglutide is no longer commercially available). ${ }^{44}$

In comparison, lixisenatide and once-weekly exenatide have only demonstrated noninferiority compared with placebo. ${ }^{47,48}$ Study investigators in the EXSCEL trial with onceweekly exenatide suggested that the disproportionate use of diabetes therapies known to reduce $\mathrm{CV}$ risk in the placebo group, such as SGLT-2is and other GLP-1RAs, may have preferentially resulted in lower event rates in the placebo arm. ${ }^{48}$

Whether there is a GLP-1RA class effect in CV safety remains to be determined, but appears unlikely given the results obtained thus far in CVOTs. As yet, the mechanism underlying the emerging cardioprotective effect of GLP$1 \mathrm{RAs}$ has not been identified and it is unknown whether this effect occurs via GLP-1, its receptor, or other pathways. ${ }^{49}$ The mixed results seen in CVOTs of GLP-1RAs may be attributed to varying molecular structures, as human analogs appear to perform better than the exendins, which are larger than, and molecularly different to, the human analogs. Further clarity will be available after the results from REWIND with dulaglutide and PIONEER 6 with oral semaglutide are published in full (NCT01394952 and NCT02692716, respectively; Clinicaltrials.gov).

Only two basal insulins have been investigated in CVOTs. Trials with insulin glargine (U100 Lantus) demonstrated no significant difference in MACE compared with SoC in the ORIGIN trial, ${ }^{50}$ and the DEVOTE trial revealed noninferiority of insulin degludec compared with insulin glargine in MACE. ${ }^{51}$

\section{The clinical implications of CVOT data for newer antidiabetic therapies}

Newer agents DPP-4is, GLP-1RAs, and SGLT-2is have the most data on CV effects. As DPP-4is do not appear to improve $\mathrm{CV}$ outcomes compared with controls, it is unlikely that they will be used to reduce $\mathrm{CV}$ benefits.
Although, real-world data have demonstrated that the benefits seen with empagliflozin in CVOTs are applicable to a broad population of patients with $\mathrm{T} 2 \mathrm{D}$ in clinical practice, ${ }^{52}$ it is uncertain how trial outcomes for other SGLT-2is will translate into the real-world setting. Additionally, safety evidence is lacking in specific populations, such as the very elderly population.

The GLP-1RAs liraglutide and semaglutide have also demonstrated a benefit in CV outcomes, ${ }^{16,45}$ which supports their use in patients at high risk of CV events. This fact has been highlighted by the ADA/EASD, who have given preference to the use of liraglutide, based on the LEADER trial, and empagliflozin, based on EMPAREG OUTCOME, in this patient population. ${ }^{11}$ Further information on the long-term effects of these agents and the mechanisms behind their functions will become available with the arrival of results from ongoing and future CVOTs with GLP-1RA and SGLT-2is. Going forward, some investigators are exploring the potential to broaden the use of newer antidiabetic therapies beyond that of T2D. The Phase III EMPEROR-Preserved and EMPERORReduced trials investigating empagliflozin in patients with chronic HF are presently ongoing (NCT03057951 and NCT03057977, respectively; Clinicaltrials.gov) and, once published, will offer insight into the potential nondiabetic use of these agents.

Both insulin glargine and insulin degludec are associated with no increased risk of CV events compared with SoC. ${ }^{50,51}$ However, weight gain and hypoglycemia remain a concern with insulin, ${ }^{53}$ and therefore other injectable therapies may be more appropriate prior to initiating insulin regimens. This is reflected in the ADA/EASD 2018 consensus report, wherein GLP-1RAs are generally recommended as the first injectable therapy. ${ }^{11}$

$\mathrm{CV}$ events are not the only adverse events collected for analysis during CVOTs. On occasion, some of these other events may indicate an unexpected safety signal. For example, in CANVAS, it was determined that there was a twofold increase in the number of lower-extremity amputations reported with canagliflozin therapy. ${ }^{15}$ This resulted in a boxed warning about the risk of lower limb amputation on the prescribing information leaflet. ${ }^{54}$ Also, SGLT-2is increase the risk of genital infections. ${ }^{14,15,38,54}$ These other adverse events indicate the need for nurse practitioners to be aware of all data reported from such studies and to discuss carefully risks and benefits with patients. Further clinical evaluation and documentation of vascular status and sensation with thorough foot exams are prudent as well. 


\section{Conclusion}

CV benefits are typically observed alongside glycemic efficacy even with older treatments, such as metformin, although no CVOTs for these treatments have been completed. Having effective glucose-lowering agents that also demonstrate multifaceted benefits such as CVD risk reduction has profound implications for the development of new agents that will, by necessity, have to be compared with strategies of care that include SGLT-2is and GLP-1RAs. Nurse practitioners have data to support making informed decisions based on the $\mathrm{CV}$ effects of different therapies and individual patient risk factors when prescribing.

For patients with T2D who are at particularly high risk for $\mathrm{CV}$ events, the $\mathrm{CV}$ risks and benefits of antidiabetic drugs should inform prescribing decisions. Currently, only empagliflozin and liraglutide are licenced for use in patients with T2D and CVD, and these therapies should be prioritized when considering treatment for patients with comorbid CVD.

\section{Acknowledgments}

The authors are grateful to Kate Booth, BSc, of Watermeadow Medical, an Ashfield company, for writing assistance in the development of this manuscript. This assistance was funded by Novo Nordisk, who also had a role in the review of the manuscript for scientific accuracy.

\section{Disclosure}

Deborah Hinnen has attended advisory boards for Janssen Pharmaceuticals, Sanofi, Eli Lilly and Co, and Novo Nordisk and has served on speakers' bureau for Eli Lilly and Co and Janssen Pharmaceuticals. Davida F Kruger has attended advisory boards for Janssen Pharmaceuticals, Abbott, Boehringer Ingelheim, Dexcom, Eli Lilly and Co, Novo Nordisk, and Sanofi Aventis; has served on speakers' bureau for Boehringer Ingelheim, Bristol-Myers Squibb, Eli Lilly and Co, Janssen Pharmaceuticals, Novo Nordisk, and Valeritas; and has received research support from Abbott, Bristol-Myers Squibb, Calibra/Johnson and Johnson, Dexcom, Eli Lilly and Co, IDC, Helmsley Charitable Trust, Lexicon, NIH, Novo Nordisk, and TEVA. The authors report no other conflicts of interest in this work.

\section{References}

1. Rawshani A, Rawshani A, Franzén S, et al. Mortality and cardiovascular disease in type 1 and type 2 diabetes. $N$ Engl J Med. 2017;376(15) 1407-1418.
2. Fox CS, Golden SH, Anderson C, et al. Update on prevention of cardiovascular disease in adults with type 2 diabetes mellitus in light of recent evidence: a scientific statement from the American Heart Association and the American Diabetes Association. Diabetes Care. 2015;38(9):1777-1803.

3. Piepoli MF, Hoes AW, Agewall S, et al. 2016 European guidelines on cardiovascular disease prevention in clinical practice. Rev Esp Cardiol. 2016;69(10):939.

4. Rao Kondapally Seshasai S, Kaptoge S, Thompson A, et al. Diabetes mellitus, fasting glucose, and risk of cause-specific death. $N$ Engl J Med. 2011;364(9):829-841.

5. International Diabetes Federation. IDF Diabetes Atlas. 8th ed; 2017 Available from: http://diabetesatlas.org/resources/2017-atlas.html. Accessed February 18, 2019.

6. Vazquez-Benitez G, Desai JR, Xu S, et al. Preventable major cardiovascular events associated with uncontrolled glucose, blood pressure, and lipids and active smoking in adults with diabetes with and without cardiovascular disease: a contemporary analysis. Diabetes Care. 2015;38(5):905-912.

7. Khaw KT, Wareham N, Luben R, et al. Glycated haemoglobin, diabetes, and mortality in men in Norfolk cohort of European Prospective Investigation of Cancer and nutrition (EPIC-Norfolk). BMJ. 2001;322(7277):15-18.

8. Stratton IM, Adler AI, Neil HA, et al. Association of glycaemia with macrovascular and microvascular complications of type 2 diabetes (UKPDS 35): prospective observational study. BMJ. 2000;321(7258): 405-412.

9. Holman RR, Paul SK, Bethel MA, Matthews DR, Neil HA. 10-year follow-up of intensive glucose control in type 2 diabetes. $N$ Engl J Med. 2008;359(15):1577-1589.

10. Inzucchi SE, Bergenstal RM, Buse JB, et al. Management of hyperglycemia in type 2 diabetes, 2015: a patient-centered approach: update to a position statement of the American Diabetes Association and the European Association for the Study of Diabetes. Diabetes Care. 2015;38(1):140-149.

11. Davies MJ, D’Alessio DA, Fradkin J, et al. Management of hyperglycemia in type 2 diabetes, 2018. A consensus report by the American Diabetes Association (ADA) and the European Association for the Study of Diabetes (EASD). Diabetes Care. 2018;41(12):2669-2701.

12. Nissen SE, Wolski K. Effect of rosiglitazone on the risk of myocardial infarction and death from cardiovascular causes. $N$ Engl J Med. 2007;356(24):2457-2471.

13. US Food and Drug Administration. Guidance for industry: diabetes mellitus - evaluating cardiovascular risk in new antidiabetic therapies to treat type 2 diabetes, 2008. Available from: www.fda.gov/downloads/Drugs/GuidanceComplianceRegulatoryInformation/Guidances/ ucm071627.pdf. Accessed February 18, 2019.

14. Zinman B, Wanner C, Lachin JM, et al. Empagliflozin, cardiovascular outcomes, and mortality in type 2 diabetes. N Engl J Med. 2015;373(22): 2117-2128.

15. Neal B, Perkovic V, Mahaffey KW, et al. Canagliflozin and cardiovascular and renal events in type 2 diabetes. NEngl J Med. 2017;377(7):644-657.

16. Marso SP, Daniels GH, Brown-Frandsen K, et al. Liraglutide and cardiovascular outcomes in type 2 diabetes. NEngl J Med.2016;375(4):311-322.

17. American Diabetes Association (ADA). Standards of medical care in diabetes. J Clin Appl Res Educ. 2017;40:142.

18. UKPDS Group. Effect of intensive blood-glucose control with metformin on complications in overweight patients with type 2 diabetes (UKPDS 34). Lancet. 1998;352(9131):854-865.

19. Monami M, Genovese S, Mannucci E. Cardiovascular safety of sulfonylureas: a meta-analysis of randomized clinical trials. Diabetes Obes Metab. 2013;15(10):938-953.

20. Home PD, Pocock SJ, Beck-Nielsen H, et al. Rosiglitazone evaluated for cardiovascular outcomes in oral agent combination therapy for type 2 diabetes (RECORD): a multicentre, randomised, open-label trial. Lancet. 2009;373(9681):2125-2135. 
21. Nissen SE. Rosiglitazone: a case of regulatory hubris. BMJ. 2013; 347:f7428.

22. Mendes D, Alves C, Batel-Marques F. Number needed to harm in the post-marketing safety evaluation: results for rosiglitazone and pioglitazone. Pharmacoepidemiol Drug Saf. 2015;24(12):1259-1270.

23. Zou C, Hu H. Use of pioglitazone in the treatment of diabetes: effect on cardiovascular risk. Vasc Health Risk Manag. 2013;9:429-433.

24. Azimova K, San Juan Z, Mukherjee D. Cardiovascular safety profile of currently available diabetic drugs. Ochsner J. 2014;14(4):616-632.

25. NAVIGATOR Study Group, McMurray JJ, Holman RR, et al. Effect of valsartan on the incidence of diabetes and cardiovascular events. N Engl J Med. 2010;362(16):1477-1490.

26. Holman RR, Coleman RL, Chan JCN, et al. Effects of acarbose on cardiovascular and diabetes outcomes in patients with coronary heart disease and impaired glucose tolerance (ACE): a randomised, double-blind, placebo-controlled trial. Lancet Diabetes Endocrinol. 2017;5(11):877-886.

27. Zhuang XD, He X, Yang DY, et al. Comparative cardiovascular outcomes in the era of novel anti-diabetic agents: a comprehensive network metaanalysis of 166,371 participants from 170 randomized controlled trials. Cardiovasc Diabetol. 2018;17(1):79.

28. Scirica BM, Bhatt DL, Braunwald E, et al. Saxagliptin and cardiovascular outcomes in patients with type 2 diabetes mellitus. $N$ Engl $J$ Med. 2013;369(14):1317-1326.

29. White WB, Pratley R, Fleck P, et al. Cardiovascular safety of the dipeptidyl peptidase- 4 inhibitor alogliptin in type 2 diabetes mellitus. Diabetes Obes Metab. 2013;15(7):668-673.

30. Green JB, Bethel MA, Armstrong PW, et al. Effect of sitagliptin on cardiovascular outcomes in type 2 diabetes. N Engl J Med. 2015;373(3): 232-242.

31. Fei Y, Tsoi MF, Kumana CR, Cheung TT, Cheung BMY. Network metaanalysis of cardiovascular outcomes in randomized controlled trials of new antidiabetic drugs. Int J Cardiol. 2018;254:291-296.

32. Cardiovascular and renal microvascular outcome study with linagliptin in patients with type 2 diabetes mellitus (CARMELINA). Presented at the European Association for the Study of Diabetes (EASD) 2018 annual meeting, Berlin, Germany; 2018. Available from: https://www. easd.org/virtualmeeting/home.html\#! contentsessions/2873. Accessed November 13, 2018.

33. Scirica BM, Braunwald E, Raz I, et al. Heart failure, saxagliptin, and diabetes mellitus: observations from the SAVOR-TIMI 53 randomized trial. Circulation. 2014;130(18):1579-1588.

34. Zannad F, Cannon CP, Cushman WC, et al. Heart failure and mortality outcomes in patients with type 2 diabetes taking alogliptin versus placebo in examine: a multicentre, randomised, double-blind trial. Lancet. 2015;385(9982):2067-2076.

35. Verma S, Goldenberg RM, Bhatt DL, et al. Dipeptidyl peptidase-4 inhibitors and the risk of heart failure: a systematic review and metaanalysis. CMAJ Open. 2017;5(1):E152-E177.

36. Wu JH, Foote C, Blomster J, et al. Effects of sodium-glucose cotransporter-2 inhibitors on cardiovascular events, death, and major safety outcomes in adults with type 2 diabetes: a systematic review and metaanalysis. Lancet Diabetes Endocrinol. 2016;4(5):411-419.

37. Lee G, Oh SW, Hwang SS, et al. Comparative effectiveness of oral antidiabetic drugs in preventing cardiovascular mortality and morbidity: a network meta-analysis. PLoS One. 2017;12(5):e0177646.

38. Boehringer Ingelheim Pharmacueticals Inc. Jardiance (empagliflozin) prescribing information. Available from: https://www.accessdata.fda. gov/drugsatfda_docs/label/2016/204629s008lbl.pdf. Accessed September 25, 2018.

39. American Diabetes Association. 8. Pharmacologic approaches to glycemic treatment: standards of medical care in diabetes-2018. Diabetes Care. 2018;41(Supplement 1):S73-S85.

40. American Diabetes Association. Standards of medical care in diabetes. Diabetes Care. 2017;40(Supplement 1):S1-S131.
41. Wiviott SD, Raz I, Bonaca MP, et al. Dapagliflozin and cardiovascular outcomes in type 2 diabets. $N$ Engl J Med. 2019;380(4):347-357.

42. Zhang X, Shao F, Zhu L, et al. Cardiovascular and microvascular outcomes of glucagon-like peptide-1 receptor agonists in type 2 diabetes: a meta-analysis of randomized controlled cardiovascular outcome trials with trial sequential analysis. BMC Pharmacol Toxicol. 2018;19(1):58.

43. Wang Q, Liu L, Gao L, Li Q. Cardiovascular safety of GLP-1 receptor agonists for diabetes patients with high cardiovascular risk: a metaanalysis of cardiovascular outcomes trials. Diabetes Res Clin Pract. 2018;143:34-42.

44. Hernandez AF, Green JB, Janmohamed S, et al. Albiglutide and cardiovascular outcomes in patients with type 2 diabetes and cardiovascular disease (HARMONY Outcomes): a double-blind, randomised placebocontrolled trial. Lancet. 2018;392(10157):1519-1529.

45. Marso SP, Bain SC, Consoli A, et al. Semaglutide and cardiovascular outcomes in patients with type 2 diabetes. NEngl J Med. 2016;375(19): 1834-1844.

46. Novo Nordisk. Victoza (liraglutide), prescribing information. 2017. Available from: https://www.accessdata.fda.gov/drugsatfda_docs/ label/2017/022341s027lbl.pdf. Accessed September 25, 2018.

47. Pfeffer MA, Claggett B, Diaz R, et al. Lixisenatide in patients with type 2 diabetes and acute coronary syndrome. N Engl J Med. 2015;373(23): 2247-2257.

48. Holman RR, Bethel MA, Mentz RJ, et al. Effects of once-weekly exenatide on cardiovascular outcomes in type 2 diabetes. NEngl J Med. 2017;377(13):1228-1239.

49. del Olmo-Garcia MI, Merino-Torres JF. GLP-1 receptor agonists and cardiovascular disease in patients with type 2 diabetes. J Diabetes Res. 2018;2018:4020492.

50. ORIGIN Trial Investigators, Gerstein HC, Bosch J, et al. Basal insulin and cardiovascular and other outcomes in dysglycemia. N Engl J Med. 2012;367(4):319-328.

51. Marso SP, McGuire DK, Zinman B, et al. Efficacy and safety of degludec versus glargine in type 2 diabetes. N Engl J Med. 2017;377(8):723-732.

52. Kosiborod M, Cavender MA, Fu AZ, et al. Lower risk of heart failure and death in patients initiated on sodium-glucose cotransporter- 2 inhibitors versus other glucose-lowering drugs: the CVD-REAL study (comparative effectiveness of cardiovascular outcomes in new users of sodiumglucose cotransporter-2 inhibitors). Circulation. 2017;136(3):249-259.

53. Russell-Jones D, Khan R. Insulin-associated weight gain in diabetes - causes, effects and coping strategies. Diabetes Obes Metab. 2007;9(6):799-812.

54. Janssen Pharmaceuticals Inc. Invokana (canagliflozin) prescribing information. Available from: http://www.janssenlabels.com/packageinsert/product-monograph/prescribing-information/INVOKANA-pi. pdf. Accessed November 09, 2018.

55. White WB, Cannon CP, Heller SR, et al. Alogliptin after acute coronary syndrome in patients with type 2 diabetes. NEngl J Med. 2013;369(14): 1327-1335.

56. Intarcia Announces Successful Cardiovascular Safety Results in Phase 3 FREEDOM-CVO Trial for ITCA 650, an Investigational Therapy for Type 2 Diabetes [press release, 2016]. Available from: https://www. intarcia.com/media/media-archive/press-releases/intarcia-announcessuccessful-cardiovascular-safety-results-in-p.html. Accessed September 25, 2018.

57. Novo Nordisk. Oral semaglutide demonstrates a favourable cardiovascular safety profile and a significant reduction in cardiovascular death and all-cause mortality in people with type 2 diabetes in the PIONEER 6 trial. [press release, 2018]. Available from: https://www.novonordisk. com/bin/getPDF.2226789.pdf. Accessed December 4, 2018.

58. Eli Lilly \& Co. Trulicity® (dulaglutide) demonstrates superiority in reduction of cardiovascular events for broad range of people with type 2 diabetes. [press release, 2018]. Available from: https://investor.lilly. com/node/39796/pdf. Accessed November 12, 2018. 
Diabetes, Metabolic Syndrome and Obesity: Targets and Therapy

Diabetes, Metabolic Syndrome and Obesity: Targets and Therapy is an international, peer-reviewed open-access journal committed to the rapid publication of the latest laboratory and clinical findings in the fields of diabetes, metabolic syndrome and obesity research Original research, review, case reports, hypothesis formation, expert opinion and commentaries are all considered for publication. The manuscript management system is completely online and includes a very quick and fair peer-review system, which is all easy to use. Visit http://www.dovepress.com/testimonials.php to read real quotes from published authors.

Submit your manuscript here: https://www.dovepress.com/diabetes-metabolic-syndrome-and-obesity-targets-and-therapy-journal 